\title{
Langanhaltende Besserung von somatischen und psychovegetativen Störungen unter Procain-Infusionen: Eine multizentrische Anwendungsbeobachtung
}

\author{
Johann Diederich Hahn-Godeffroy ${ }^{a}$ Simona Mangold ${ }^{b} \quad$ Monika Bernert $^{c} \quad$ Annette Bartelt $^{\mathrm{d}}$ \\ Thomas Herdegen ${ }^{\mathrm{d}}$ \\ ${ }^{a}$ Facharzt für Pharmakologie und Innere Medizin, Hamburg, Deutschland; \\ ${ }^{b}$ Fachärztin für Allgemeinmedizin, Laupheim, Deutschland; \\ ${ }^{c}$ Fachärztin für Allgemeinmedizin, Usingen, Deutschland; \\ dInstitut für Experimentelle und Klinische Pharmakologie, Universitätsklinikum Schleswig-Holstein, Campus Kiel, Kiel, Deutschland
}

\section{Schlüsselwörter}

Befindlichkeitsskalierungsfragebogen · "Drug repurposing» · Infusion · Procain · Psychovegetative Störungen

\section{Zusammenfassung}

Hintergrund: Procain besitzt wie das strukturverwandte Cocain zentralnervöse Wirkungen unabhängig von seinem lokalanästhetischen Effekt. Procain-Infusionen werden Patienten mit psychovegetativen Störungen seit Jahrzehnten verabreicht, zumeist im Rahmen einer Neuraltherapie. Untersuchungen zur Wirksamkeit einer alleinigen Gabe von Procain im Praxisalltag stehen aus. Patienten und Methoden: Im Rahmen einer Anwendungsbeobachtung (AWB) wurde bei 56 Patienten aus 3 Arztpraxen die Wirkung von intravenösen Procain-Infusionen (1-3 Ampullen à $5 \mathrm{ml}$ einer $2 \%$ igen Procain-Lösung in $250 \mathrm{ml}$ Natriumchlorid pro Arztbesuch) auf das somatische und psychovegetative Befinden dokumentiert. Hierzu wurde ein validierter, 21 Fragen umfassender Befindlichkeitsskalierungsfragebogen verwendet, den die Patienten vor Beginn und nach 1, 2, 4 und 6 Monaten ausfüllten. Neben der laufenden Medikation waren keine weiteren therapeutischen Maßnahmen erlaubt. Ergebnisse: Nach 4 oder 6 Monaten zeigten 42 Patienten (75\%) eine Verbesserung bei den 9 positiven Items (d.h. erfragte Eigenschaften) wie "Freude», "Genussfähigkeit» oder "Schlaf»; 35 Patienten (62,5\%) verbesserten sich bei den 12 negativen Items wie "Stressbelastung", "Energielosigkeit» oder "Ängstlichkeit». Diese Veränderungen waren nach 2, 4 und 6 Monaten signifikant gegenüber dem Ausgangswert. Schlussfolgerungen: Procain ruft im zentralen Nervensystem nachweislich Veränderungen in der Aktivität spezifischer Kerngebiete wie des limbischen und kortikalen Systems hervor. Die Ergebnisse dieser AWB deuten auf eine langanhaltende Verbesserung von somatischen und psychovegetativen Störungen unter der Infusion von Procain.

(c) 2018 S. Karger AG, Basel
Keywords

Mental-state scaling questionnaire - Drug repurposing . Infusion · Procaine - Psycho-vegetative disturbances

\section{Summary}

Longlasting Improvement of Somatic and Psycho-

Vegetative Disturbances under Procaine Infusions:

A Case-Control Series

Background: As with the structurally related cocaine, procaine exerts effects in the central nervous system beyond its local anesthetic actions. For decades, procaine infusions have been applied in patients with psycho-vegetative disturbances, mostly during neural therapy. The efficacy of procaine infusions alone under real-life conditions has not been studied so far. Patients and Methods: In this case control series from 3 medical practices, the effect of intravenous procaine (1-3 ampoules à $5 \mathrm{ml}$ of procaine $2 \%$ in $250 \mathrm{ml}$ sodium chloride per treatment setting) on the somatic and psycho-vegetative state of health was documented in 56 patients. Thereto, the patients answered a validated German mental-state scaling questionnaire with 21 questions at the beginning and after 1, 2, 4, and 6 months. Apart from their current medication, no further pharmacotherapeutic interventions were allowed. Results: After 4 or 6 months, 42 patients (75\%) showed an improvement in the 9 positive items, e.g., 'hedonia', 'joyness', or 'improved sleep'. 35 Patients (62,5\%) reported a substantial attenuation in the 12 negative items, e.g., 'stress reactions', 'loss of energy', or 'anxiety'. All these changes were significant after 2, 4, and 6 months compared to the values at baseline. Conclusions: The results of this case-control series suggest a lasting improvement of somatic and psychovegetative symptoms under the infusion of procaine alone, which modulates the activities of specific brain areas such as the limbic system.

\section{KARGER}

(c) 2018 S. Karger AG, Basel
Prof. Dr. med. Thomas Herdegen 


\section{Einleitung}

Patienten und behandelnde Ärzten berichten häufig, dass intravenös verabreichtes Procain in Form eines erlebten therapeutischen Effekts psychovegetative und/oder somatische Störungen verbessert. Das initiale Schlüsselereignis war eine versehentliche intravenöse Gabe von Procain, mit der der Düsseldorfer Arzt Ferdinand Huneke die Migräne und den begleitenden erheblichen depressiven Verstimmungszustand seiner Schwester beseitigen konnte (Übersichten bei [1-3]).

Unabhängig von seiner lokalanästhetischen Wirkung und der Einbindung in therapeutische Konzepte wie die Basen- oder Neuraltherapie ruft Procain eigenständige neurobiologische Effekte hervor. Im Tierversuch, einschließlich Primaten, wurde die Bindung von Procain unter anderem an muskarinerge AcetylcholinRezeptoren sowie an den Dopamin-Wiederaufnahmetransporter (DAT) nachgewiesen [4-6]. Am Menschen verursacht die intravenöse Gabe von Procain Änderungen der zerebralen Durchblutung, etwa im Kortex und im limbischen System, und verändert die Stimmungslage [7-9]; manche dieser Procain-Wirkungen ähneln denen von Cocain, allerdings in einem schwächeren Ausmaß.

Die Auswirkung alleiniger auf Wochen oder Monate angelegter Infusion von Procain auf das körperliche und psychische Allgemeinbefinden war bisher nicht Gegenstand von wissenschaftlichen Untersuchungen. Daher wurde hier zum ersten Mal der Versuch unternommen, mittels eines validierten Befindlichkeitsfragebogens und eines definierten Protokolls die langfristige Wirkung von Procain multizentrisch zu dokumentieren. Eine solche primäre Anwendungsbeobachtung (AWB) ist umso notwendiger, da die alleinige Procain-Infusion unabhängig von anderen Therapiekonzepten immer häufiger in der ärztlichen Praxis Anwendung findet. Schließlich wird das «drug repurposing» bzw. «rescue» und «repositioning» (DRRR) von altbekannten Arzneistoffen immer wichtiger, gerade in Bezug auf neuro-psychische Störungen, für die keine neuen Arzneistoffe in Sicht sind.

Die vorliegende AWB legt nahe, dass sich die Beschwerden von bis zu zwei Dritteln der Patienten mit affektiven Störungen, Stressfolgeerkrankungen oder chronischen Schmerzsymptomen unter Infusionsbehandlungen mit Procain bessern.

\section{Protokoll der Anwendungsbeobachtung und statistische Methoden}

\section{Durchführung der Befragung}

Diese AWB folgte einem unkontrollierten Ansatz; sie war offen, nichtrandomisiert und parallel. Die AWB begann jeweils ab einem von jeder Praxis selbst festgelegten Stichtag. Alle Patienten, die auch ohne diese AWB eine ProcainInfusion erhalten hätten, wurden ohne weitere Selektionierung und unabhängig von der Diagnose um ihre Teilnahme gebeten und unter Berücksichtigung der Ein- und Ausschlusskriterien nach ihrer Zustimmung eingeschlossen.

\section{Fragebogen}

Der BSKE (BSKE = Befindlichkeitsskalierungsfragebogen) zur körperlichen und seelischen Befindlichkeit wurde von Prof. Dr. W. Janke (Institut für
Medizinische Psychologie, Universität Würzburg) entwickelt und als Instrument für verschiedene Untersuchungen und Studien eingesetzt und validiert $[10,11]$.

Das primäre Zielkriterium war die explorative, deskriptive Beschreibung der Veränderung des positiven und negativen BSKE-Summenscores (Tab. 1) vom Beobachtungsbeginn bis 6 Monate danach. Die Patienten hatten insgesamt 5 Fragebögen auszufüllen: vor der ersten Procain-Infusion (Ausgangwert, Fragebogen bzw. Beobachtungszeitpunkt 0), nach 1, 2, 4 und 6 Monaten (Fragebogen bzw. Beobachtungszeitpunkte 1, 2, 4 und 6).

Zur Auswertung des BSKE wurde aus den Skalen mit den positiven und negativen Items je ein Summenscore gebildet. Die Veränderungen dieser beiden Summenscores wurden jeweils getrennt statistisch berechnet. Die Fragen des BSKE umfassten:

- 9 positive Items (je 0-6 Punkte, insgesamt 54 Punkte; je höher die Punktzahl, desto besser das Befinden): seelisches Wohlbefinden, Selbstsicherheit, Aktivität, gehobene Stimmung, innere Entspannung, Konzentriertheit, Wachheit, Genussfähigkeit, Freude;

- 12 negative Items (je 0-6 Punkte, insgesamt 72 Punkte; je niedriger die Punktzahl, desto besser das Befinden): innere Erregtheit, Schmerzbelastung, Energielosigkeit, Benommenheit, Ängstlichkeit, Ärger, Aggressivität, Müdigkeit, Missstimmung, Stressbelastung, Feindseligkeit, Traurigkeit.

\section{Einschlusskriterien}

Als Einschlusskriterien galten grundsätzlich alle Erkrankungen, für die die Ärzte eine Procain-Infusion anboten, wie psychovegetative Störungen bei chronischen Erkrankungen z.B. im Rahmen von affektiven Störungen wie depressiver Verstimmung oder Burn-out-Symptomen, Symptome einer aktivierten Stressachse, Schlaflosigkeit oder Unruhe, diffuse unspezifische Schmerzen, Verdauungsstörungen oder gastrointestinale Symptome, außerdem die Ablehnung oder Erfolglosigkeit etablierter Therapiekonzepte.

\section{Ausschluss- und Abbruchkriterien}

Die Ausschluss- und Abbruchkriterien waren ein Alter unter 18 Jahren, akute Erkrankungen und Erkrankungen und Störungen, bei denen eine leitlinienkonforme Therapie erwiesenermaßen eine Besserung bewirken würde. Hiervon ausgenommen waren Fälle, in denen eine solche Therapie vom Patienten abgelehnt wurde oder definitiv erfolglos geblieben war (siehe oben) und in denen Procain gleichzeitig anderweitig angewendet wurde.

\section{Aufklärung und Einwilligung}

Die Patienten wurden, wie vor jeder Behandlung mit Procain, zunächst über eine - sofern verfügbar - leitliniengerechte Therapie und über die möglichen Risiken einer Procain-Infusion mündlich aufgeklärt.

Für diese Untersuchung wurden die Patienten zusätzlich über die Verwendung des Fragebogens und der Daten für eine AWB aufgeklärt. Auch auf das Recht, zu jeder Zeit ohne Angabe von Gründen die Procain-Therapie und/oder die Beantwortung des Fragebogens abzubrechen, wurden die Patienten hingewiesen. Alle Patienten mussten schriftlich ihre Einwilligung erklären und die Aufklärungen bestätigen.

\section{Dosierung und Applikationsintervall}

Die Dosierung und Applikationshäufigkeit richteten sich nach individueller, freier ärztlicher Therapieplanung. Zu Beginn wurden 1-2-mal pro Woche im Abstand von 1 oder 2 Wochen jeweils 1-2, seltener 3, Ampullen à $5 \mathrm{ml}$ einer $2 \%$ igen Procain-Lösung in $250 \mathrm{ml}$ Natriumchlorid $(\mathrm{NaCl})$ pro Behandlungssitzung verabreicht. Mit zunehmender Behandlungsdauer und/oder Symptomverbesserung nahm die Zahl der Sitzungen ab. Die Teilnahme an der AWB hatte keinen Einfluss auf die sonstige Begleittherapie.

\section{Ethische Standards}

Die vorliegende AWB wurde in Übereinstimmung mit den CARE-Guidelines $(\mathrm{CARE}=$ Case Report) durchgeführt. Alle 3 Ethikkommissionen der Landesärztekammern, in deren Bereichen die AWB durchgeführt wurde, wurden über diese informiert (Universal Trial Number U1111-1184-5341). 
Tab. 1. Gesamtscore der positiven Items

\begin{tabular}{|c|c|c|c|c|c|}
\hline & \multicolumn{5}{|l|}{ Monat } \\
\hline & 0 & 1 & 2 & 4 & 6 \\
\hline Mittelwert \pm SD & $24,0 \pm 8,1$ & $33,4 \pm 7,6$ & $35,9 \pm 7,6$ & $34,0 \pm 8,5$ & $36,5 \pm 8,4$ \\
\hline Ausgefüllte Fragebögen, n & 56 & 54 & 35 & 39 & 48 \\
\hline Signifikanz gegenüber Monat 0 & & $<0,00001$ & $<0,00001$ & $<0,00001$ & $<0,00001$ \\
\hline Signifikanz gegenüber Monat 1 & & & 0,037 & 0,22 & 0,0016 \\
\hline Effektstärke ${ }^{\star}$ gegenüber Monat 0 & & 0,55 & 0,57 & 0,58 & 0,59 \\
\hline 95\%-Konfidenzintervall & & 7 bis 11,5 & 8,5 bis 15 & 7,5 bis 13 & 10 bis 15,5 \\
\hline Mediane Differenz & & 9 & 11,5 & 10 & 13 \\
\hline
\end{tabular}

${ }^{*}$ Nach Rosenthal [14]: 0,1: geringer Effekt; 0,3: mittlerer Effekt; 0,5: großer Effekt.
Die Ethikkommissionen stimmten dem Studiendesign und der biometrischen Planung zu. Diese AWB erfüllt die Kriterien der STROBE-Guidelines $(\mathrm{STROBE}=$ Strengthening the Reporting of Observational Studies in Epidemiology) [12].

\section{Biometrische Planung}

Eine biometrische Fallzahlplanung war aufgrund fehlender Literatur zur alleinigen Gabe von intravenösem Procain schwierig. Ausgehend davon, dass eine Effektstärke von $\mathrm{d}=0,5$ vorlag (eine Veränderung um 1 Punkt eines Subscores wird als klinisch signifikant angesehen; Standardabweichung = 1,8 [13]) und unter der Vorgabe von $\mathrm{p}=0,05$ und Power $=95 \%$ wurde für den 2-seitigen, verbundenen Mann-Whitney-U-Test eine Gesamtgruppengröße von mindestens 56 Patienten errechnet.

Auswertungen und Statistik

Die anonymisierten Fragebögen wurden von einer praxisfernen Person außerhalb der Praxis ausgewertet.

\section{Anforderung an eine gültige Auswertung}

Jeder Teilnehmer musste mindestens an 4 von 5 Zeitpunkten den Fragebogen ausgefüllt haben. Zudem musste der Fragebogen mindestens nach 4 oder 6 Monaten ausgefüllt worden sein.

\section{Auswertung des Verlaufs}

Die Gesamtpunktzahl aller positiven bzw. aller negativen Items (Tab. 1) wurde für jeden Patienten pro Zeitpunkt errechnet. Mittels BiAS-Software wurde mit einem «Wilcoxon-matched-pair»-Test die Signifikanz der Differenz zwischen dem Ausgangswert 0 und den Beobachtungszeitpunkten 1 (nach 1 Monat), 2 (nach 2 Monaten), 4 (nach 4 Monaten) und 6 (nach 6 Monaten) bestimmt. Die Differenz wurde bei p-Werten $<0,05$ als signifikant bewertet Der berechnete Hodges-Lehmann-Schätzer zeigte den Median der Differenzen zwischen dem Ausgangswert und dem jeweiligen Beobachtungsmonat. Das anhand dieses Werts bestimmte Tukey-Konfidenzintervall wurde so gewählt, dass der errechnete Wert mit einer 95\%igen Wahrscheinlichkeit in diesem Intervall lag.

\section{Berechnung der Effektstärke}

Mit der BiAS-Software wurde auch die Effektstärke nach Rosenthal nach der Formel $r=Z / \operatorname{sqr}\left(2^{\star} n^{c}\right)$ berechnet. Hierbei bedeutet ein Wert von 0,1 einen geringen Effekt und ein Wert von 0,5 einen großen Effekt. Einen weiteren Hinweis auf die Effektstärke geben die Konfidenzintervalle [14].

Definition einer Response

Zusätzlich wurde die Zahl der Responder (= Therapie hat angeschlagen) bestimmt. Als Response galt ein Gesamtscore, der im Vergleich zum Ausgangswert (Beobachtungszeitpunkt 0) bei 2 der 3 Zeitpunkte 2, 4 und 6 Monate größer als +7 (positive Items) bzw. größer als -10 (negative Items) war. Wir gehen davon aus, dass diese Unterschiede ( +7 und -10$)$ einer wirksamen Effektstärke entsprechen.
Definition des Response-Werts

Der Response-Wert ist der Mittelwert, der sich aus den beiden besten Differenzwerten der Zeitpunkte 2, 4 oder 6 Monate jeweils minus den Ausgangswert ergibt.

\section{Ergebnisse}

Die behandelnden Ärzte baten diejenigen Patienten um eine Teilnahme an der AWB, die aufgrund der langjährigen ärztlichen Erfahrung möglicherweise auf Procain-Infusionen ansprechen könnten. Übergreifend über die 3 Arztpraxen, die in Nord-, Mittelund Süddeutschland liegen, zeigten fast alle Patienten affektive Störungen oder Schlafstörungen; ungefähr ein Drittel der Patienten litt (auch) an chronischen Schmerzsymptomen.

\section{Auswertung der Fragebögen}

Von den 56 Teilnehmern füllten 8 den Fragebogen zu allen 5 Zeitpunkten aus. 48 Teilnehmer füllten 4 von 5 Fragebögen aus. Die Beobachtungsdauer von 6 Monaten erreichten 48 Patienten.

\section{Positive Items}

Gesamtsumme der Skala

Vor der ersten Procain-Infusion betrug der Mittelwert aller 9 positiven Items pro Patient $24,0 \pm 8,1$ (Tab. 1). Innerhalb von 4 Wochen erhöhte sich der positive Gesamtscore auf 33,4 $\pm 7,6$, erreichte mit 35,9 \pm 7,6 einen ersten maximalen Wert nach 2 Monaten und verblieb mit 36,5 $\pm 8,4$ bis zum Ende der Beobachtungszeit auf diesem erhöhten Niveau.

Dabei waren die Veränderungen im Vergleich zur initialen $\mathrm{Er}-$ hebung nach 1, 2, 4 und 6 Monaten signifikant, aber nicht zwischen den Zeitpunkten (Tab. 1). Nach dem 2. Monat war bereits ein submaximaler Gesamtwert erreicht, der sich nach 4 oder 6 Monaten nicht mehr signifikant verbesserte. Die Effektstärke gegenüber dem Ausgangswert war bei 3 von 4 Beobachtungszeitpunkten groß $(>0,5)$. Je höher der Ausgangswert lag, desto geringer war die weitere Verbesserung zwischen Ausgangswert und Endwert: Sie betrug bei einem Ausgangswert von 31-40 Gesamtpunkten +7,2 $(\mathrm{p}=0,008)$ und bei einem Ausgangswert von 11-20 Gesamtpunkten $+16,6(\mathrm{p}=0,00002)$. 
Abb. 1. Verlauf ausgewählter positiver und negativer Items: Die Abbildung zeigt den Verlauf des Gesamtscores (Mittelwert aus allen Fragebögen) der positiven Items «Freude» und «Wachheit» sowie der negativen Items «Energielosigkeit» und «Missstimmung». Die Standardabweichungen sind in Tabelle 2 und $6 \mathrm{zu}$ finden.

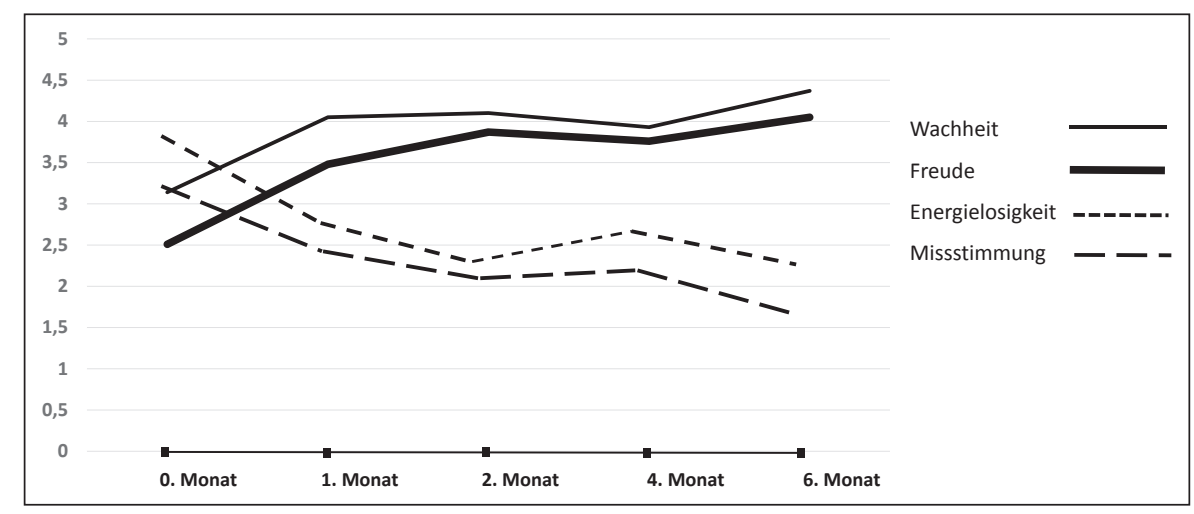

Tab. 2. Die einzelnen positiven Items

\begin{tabular}{llllll}
\hline & \multicolumn{5}{l}{ Monat } \\
\cline { 2 - 5 } & 0 & 1 & 2 & 4 & 6 \\
\hline 2. Seelisches Wohlbefinden & $2,7 \pm 1,2$ & $3,8 \pm 1,1$ & $3,9 \pm 1,1$ & $3,8 \pm 1,3$ & $4,2 \pm 1,2$ \\
6. Aktivität & $2,8 \pm 1,2$ & $3,7 \pm 1,1$ & $4,3 \pm 1,0$ & $3,8 \pm 1,4$ & $3,9 \pm 1,1$ \\
7. Innere Entspannung & $2,1 \pm 1,1$ & $3,4 \pm 1,3$ & $3,9 \pm 1,2$ & $3,6 \pm 1,2$ & $3,7 \pm 1,3$ \\
9. Wachheit & $3,2 \pm 1,3$ & $4,1 \pm 0,9$ & $4,0 \pm 1,1$ & $4,0 \pm 1,1$ & $4,4 \pm 0,8$ \\
10. Gefühl der Freude & $2,6 \pm 1,3$ & $3,6 \pm 1,1$ & $3,9 \pm 1,1$ & $3,8 \pm 1,0$ & $4,1 \pm 1,0$ \\
12. Selbstsicherheit & $2,9 \pm 1,4$ & $3,8 \pm 1,0$ & $3,9 \pm 0,9$ & $3,7 \pm 1,1$ & $4,0 \pm 1,1$ \\
17. Gehobene Stimmung & $2,5 \pm 1,3$ & $3,5 \pm 1,0$ & $3,7 \pm 1,1$ & $3,7 \pm 1,1$ & $3,8 \pm 1,3$ \\
18. Konzentriertheit & $2,6 \pm 1,4$ & $3,6 \pm 1,2$ & $3,9 \pm 1,0$ & $3,8 \pm 1,2$ & $4,1 \pm 1,1$ \\
20. Genießen-Können & $3,0 \pm 1,6$ & $3,9 \pm 1,2$ & $4,3 \pm 1,1$ & $3,9 \pm 1,3$ & $4,4 \pm 1,3$ \\
\hline
\end{tabular}

Nummerierung und Reihenfolge wie im Befindlichkeitsskalierungsfragebogen (Mittelwert \pm Standardabweichung).

\begin{tabular}{llc}
\hline Positive Items & Differenz Monat 0 und Monat 6 & Differenz Monat 2 und Monat 6 \\
\hline Innere Entspannung & 1,56 & $-0,17$ \\
Konzentriertheit & 1,47 & 0,17 \\
Gefühl der Freude & 1,47 & 0,14 \\
Seelisches Wohlbefinden & 1,46 & 0,21 \\
Genießen-Können & 1,39 & 0,13 \\
Gehobene Stimmung & 1,37 & 0,14 \\
Aktivität & 1,17 & $-0,34$ \\
Wachheit & 1,15 & 0,35 \\
Selbstsicherheit & 1,09 & 0,11 \\
\hline
\end{tabular}

Die Items sind nach der Stärke der Veränderung zwischen dem Ausgangswert von Monat 0 bzw. Monat 2 und dem Wert von Monat 6 angeordnet, beginnend mit der stärksten Veränderung (= beste Response) zwischen dem Ausgangswert zu Monat 0 und Monat 6 .
Tab. 3. Differenzen der Mittelwerte der einzelnen positiven Items

\section{Responder}

Von 56 Patienten zeigten 42 (75\%) eine Verbesserung $\geq 7$ Punkten bei dem Vergleich des Anfangswerts mit dem Mittelwert der beiden besten Werte nach 2, 4 oder 6 Monaten.

\section{Einzelne positive Items}

Generell nahm die Punktzahl der einzelnen Items entsprechend dem Gesamtscore zu (Abb. 1, Tab. 2); alle Items verbesserten sich zwischen dem 0 . und 6. Monat signifikant $(\mathrm{p}<0,01)$. Im Vergleich der Differenzen der Mittelwerte der jeweiligen Einzelitems zwischen dem 0 . und 6. Monat zeigten sich die größten Verbesserun- gen bei «Innerer Entspannung» (im Mittel +1,6 Punkte), «Gefühl der Freude», «Konzentriertheit» und «Seelisches Wohlbefinden» (im Mittel +1,5 Punkte) (Tab. 3).

\section{Vergleich der Praxen}

Der Verlauf der Skala der positiven Items mit einer submaximalen Besserung nach 2 Monaten und Persistenz bis nach 6 Monaten war in allen 3 Praxen ähnlich (Abb. 2, Tab. 4). Die Besserungen zwischen dem 0. und 6. Monat betrugen im Mittel zwischen 10,7 (Praxis 3) und 13,5 Punkte (Praxis 1). 
Abb. 2. Verlauf der Gesamtscores aller positiven Items der 3 Praxen. Jede Linie zeigt den Verlauf des Mittelwerts der Gesamtpunktzahl aller positiven Items aller Patienten einer Praxis über 6 Monate. Die dicke Linie repräsentiert den Durchschnitt der 3 Praxen. Die maximal mögliche Punktzahl betrug 54 (9 Items à 6 Punkte).

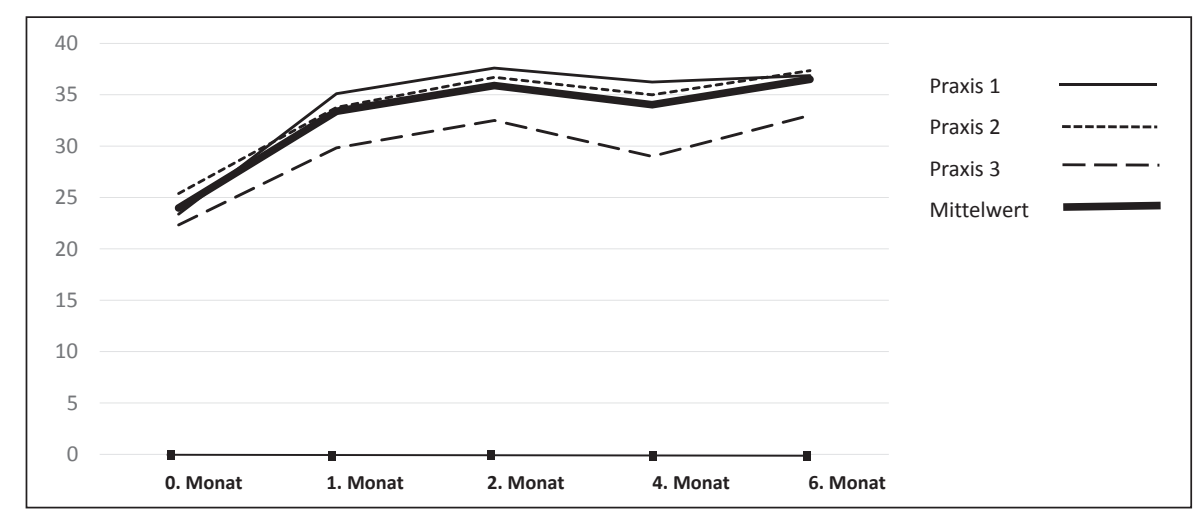

Tab. 4. Gesamtscore der positiven Items der einzelnen Praxen (Mittelwert \pm Standardabweichung)

\begin{tabular}{|c|c|c|c|c|c|}
\hline & \multicolumn{5}{|l|}{ Monat } \\
\hline & 0 & 1 & 2 & 4 & 6 \\
\hline Praxis 1 & $23,4 \pm 8,1$ & $35,1 \pm 7,3$ & $37,6 \pm 7,0$ & $36,2 \pm 7,8$ & $36,9 \pm 8,9$ \\
\hline Praxis 2 & $25,4 \pm 9,0$ & $33,8 \pm 6,3$ & $36,7 \pm 6,0$ & $35,0 \pm 7,7$ & $37,4 \pm 6,7$ \\
\hline Praxis 3 & $22,3 \pm 5,8$ & $29,8 \pm 9,7$ & $32,5 \pm 9,4$ & $29,0 \pm 9,7$ & $33,0 \pm 11,2$ \\
\hline Mittelwert & $24,0 \pm 8,1$ & $33,4 \pm 7,6$ & $35,9 \pm 7,6$ & $34,0 \pm 8,5$ & $36,5 \pm 8,4$ \\
\hline
\end{tabular}

Tab. 5. Gesamtscore der negativen Items

Tab. 6. Die einzelnen negativen Items

\begin{tabular}{llllll}
\hline & \multicolumn{7}{l}{ Monat } & & \\
\cline { 2 - 6 } & 0 & 1 & 2 & 4 & 6 \\
\hline Mittelwert \pm Standardabweichung & $33,5 \pm 12,4$ & $24,2 \pm 11,5$ & $22,8 \pm 11,5$ & $23,1 \pm 11,7$ & $19,4 \pm 10,9$ \\
Ausgefüllte Fragebögen, n & 56 & 54 & 35 & 39 & 48 \\
Signifikanz gegenüber Monat 0 & & $<0,00001$ & 0,000024 & $<0,00001$ & $<0,00001$ \\
Signifikanz gegenüber Monat 1 & & 0,39 & 0,42 & 0,0019 \\
Effektstärke* gegenüber Monat 0 & & 0,52 & 0,47 & 0,52 & 0,57 \\
95\%-Konfidenzintervall & $-11,5$ bis $-6,5$ & 14 bis -6 & $-15,5$ bis $-7,5$ & 16,5 bis -10 \\
Mediane Differenz & -9 & -10 & $-11,5$ & $-13,5$ \\
\hline
\end{tabular}

${ }^{*}$ Nach Rosenthal [14]: 0,1: geringer Effekt; 0,3: mittlerer Effekt; 0,5: großer Effekt.

\begin{tabular}{|c|c|c|c|c|c|}
\hline & \multicolumn{5}{|l|}{ Monat } \\
\hline & 0 & 1 & 2 & 4 & 6 \\
\hline 1. Innere Erregtheit & $3,3 \pm 1,7$ & $2,2 \pm 1,4$ & $2,4 \pm 1,4$ & $2,1 \pm 1,3$ & $1,7 \pm 1,3$ \\
\hline 3. Energielosigkeit & $3,6 \pm 1,8$ & $2,5 \pm 1,4$ & $2,0 \pm 1,4$ & $2,3 \pm 1,4$ & $2,0 \pm 1,3$ \\
\hline 4. Ängstlichkeit & $2,6 \pm 1,7$ & $1,7 \pm 1,5$ & $1,8 \pm 1,4$ & $1,5 \pm 1,4$ & $1,4 \pm 1,3$ \\
\hline 5. Aggressivität & $2,3 \pm 1,8$ & $1,5 \pm 1,3$ & $1,9 \pm 1,3$ & $1,3 \pm 1,3$ & $1,2 \pm 1,3$ \\
\hline 8. Missstimmung & $2,8 \pm 1,6$ & $2,0 \pm 1,3$ & $1,7 \pm 1,2$ & $1,8 \pm 1,2$ & $1,2 \pm 1,1$ \\
\hline 11. Traurigkeit & $2,9 \pm 1,7$ & $2,0 \pm 1,4$ & $1,9 \pm 1,4$ & $1,8 \pm 1,5$ & $1,5 \pm 1,4$ \\
\hline 13. Schmerzbelastung & $3,3 \pm 1,9$ & $2,6 \pm 1,6$ & $2,4 \pm 1,6$ & $2,8 \pm 1,5$ & $2,6 \pm 1,6$ \\
\hline 14. Benommenheit & $2,0 \pm 1,7$ & $1,7 \pm 1,4$ & $1,5 \pm 1,4$ & $1,4 \pm 1,4$ & $1,0 \pm 1,0$ \\
\hline 15. Ärger & $2,5 \pm 1,6$ & $1,7 \pm 1,2$ & $1,7 \pm 1,3$ & $1,7 \pm 1,3$ & $1,4 \pm 1,2$ \\
\hline 16. Müdigkeit & $3,3 \pm 1,7$ & $2,6 \pm 1,4$ & $2,1 \pm 1,2$ & $2,5 \pm 1,4$ & $2,1 \pm 1,4$ \\
\hline 19. Stress-Belastung & $3,4 \pm 1,8$ & $2,5 \pm 1,3$ & $2,5 \pm 1,2$ & $2,6 \pm 1,3$ & $2,3 \pm 1,5$ \\
\hline 21. Feindseligkeit & $2,0 \pm 1,5$ & $1,6 \pm 1,5$ & $1,1 \pm 1,1$ & $1,3 \pm 1,3$ & $1,4 \pm 1,5$ \\
\hline
\end{tabular}

Nummerierung und Reihenfolge wie im Fragebogen (Mittelwert \pm Standardabweichung). 
Tab. 7. Differenzen der Mittelwerte der einzelnen negativen Items
Tab. 8. Gesamtscore der negativen Items der einzelnen Praxen (Mittelwert \pm Standardabweichung)

\begin{tabular}{llc}
\hline Negative Items & Differenz Monat 0 und Monat 6 & Differenz Monat 2 und Monat 6 \\
\hline Innere Erregtheit & $-1,61$ & $-0,71$ \\
Missstimmung & $-1,59$ & $-0,45$ \\
Energielosigkeit & $-1,52$ & 0,03 \\
Traurigkeit & $-1,32$ & $-0,3$ \\
Müdigkeit & $-1,21$ & $-0,01$ \\
Ängstlichkeit & $-1,17$ & $-0,45$ \\
Ärger & $-1,13$ & $-0,36$ \\
Stress-Belastung & $-1,1$ & $-0,15$ \\
Benommenheit & $-1,06$ & $-0,51$ \\
Aggressivität & $-1,02$ & $-0,66$ \\
Schmerzbelastung & $-0,67$ & 0,23 \\
Feindseligkeit & $-0,66$ & 0,24 \\
\hline
\end{tabular}

Die Items sind nach der Stärke der Veränderung zwischen dem Ausgangswert von Monat 0 bzw. Monat 2 und dem Wert von Monat 6 angeordnet, beginnend mit der stärksten Veränderung (= beste Response) zwischen dem Ausgangswert zu Monat 0 und Monat 6.

\begin{tabular}{llllll}
\hline & \multicolumn{1}{l}{ Monat } & & & & \\
\cline { 2 - 6 } & 0 & 1 & 2 & 4 & 6 \\
\hline Praxis 1 & $29,7 \pm 10,2$ & $19,7 \pm 9,0$ & $18,9 \pm 10,3$ & $18,9 \pm 11,7$ & $16,0 \pm 10,7$ \\
Praxis 2 & $36,2 \pm 13,3$ & $25,4 \pm 11,5$ & $22,3 \pm 10,8$ & $22,9 \pm 11,5$ & $20,9 \pm 9,7$ \\
Praxis 3 & $35,1 \pm 13,2$ & $29,5 \pm 13,3$ & $29,2 \pm 12,2$ & $29,6 \pm 10,0$ & $25,7 \pm 12,7$ \\
Mittelwert & $33,5 \pm 12,4$ & $24,2 \pm 11,5$ & $22,8 \pm 11,5$ & $23,1 \pm 11,7$ & $19,4 \pm 10,9$ \\
\hline
\end{tabular}

Abb. 3. Verlauf der Gesamtscores der negativen Items der 3 Praxen. Jede Linie zeigt den Verlauf des Mittelwerts der Gesamtpunktzahl aller negativen Items aller Patienten einer Praxis über 6 Monate. Die dicke Linie repräsentiert den Durchschnitt der 3 Praxen. Die maximal mögliche Punktzahl betrug 72 (12 Items à 6 Punkte).

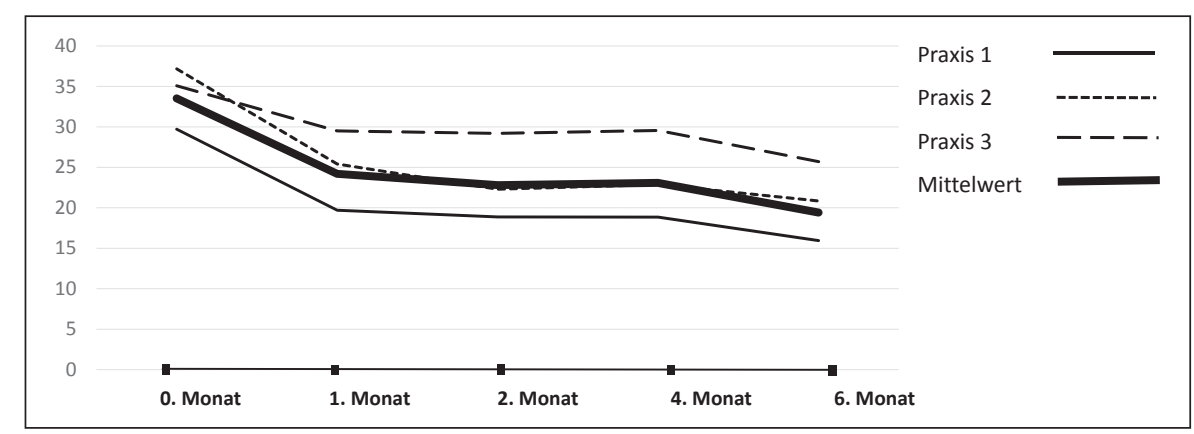

\section{Negative Items}

\section{Gesamtsumme der Skala}

Vor der ersten Procain-Infusion betrug der Mittelwert aller 12 negativen Items 33,5 $\pm 12,9$ (Tab. 5). Innerhalb von 4 Wochen nahm der negative Gesamtscore auf 24,2 $\pm 11,5 \mathrm{ab}$, verminderte sich auf ein erstes Minimum von 22,8 \pm 11,5 nach 2 Monaten und verblieb mit 19,4 \pm 10,9 auf diesem niedrigen Niveau bis zum Ende der Beobachtungszeit. Dabei war die Veränderung im Vergleich zur initialen Erhebung nach 1, 2, 4 und 6 Monaten signifikant (Tab. 5), zwischen den Zeitpunkten aber nicht (Tab. 5). Nach dem 2. Monat war bereits ein submaximaler Gesamtwert erreicht, der sich nach 4 oder 6 Monaten nicht mehr signifikant verbesserte. Die Effektstärke gegenüber dem Ausgangswert war bei allen 4 Beobachtungszeitpunkten groß $(>0,5)$. Je höher der Ausgangswert lag, desto ausgeprägter war die weitere Verbesserung zwischen Ausgangswert und Endwert.
Responder

Von 56 Patienten zeigte 35 (62,5\%) eine Verbesserung bzw. Abnahme des negativen Gesamtscores $\geq 10$ Punkte bei dem Vergleich des Anfangswerts mit dem Mittel der beiden besten Werte nach 2, 4 oder 6 Monaten.

\section{Einzelne negative Items}

Generell nahm die Punktzahl der einzelnen Items entsprechend dem Gesamtscore ab (Tab. 6); alle Items verbesserten sich zwischen dem 0 . und 6. Monat signifikant $(\mathrm{p}<0,01)$. Im Vergleich der Differenzen der Mittelwerte der jeweiligen Einzelitems zwischen dem 0. und 6. Monat zeigten sich die größten Verbesserungen bei «Missstimmung» und «Innere Erregtheit» (im Mittel -1,6 Punkte) sowie bei «Energielosigkeit» (im Mittel -1,5 Punkte) (Abb. 1, Tab. 7). 


\section{Vergleich der Praxen}

Der Verlauf der negativen Items war in allen 3 Praxen mit einer submaximalen Besserung nach 2 Monaten und Persistenz bis nach 6 Monaten ähnlich (Abb. 3, Tab. 8). Die Besserungen zwischen dem 0. und 6. Monat betrugen im Mittel zwischen 9,4 (Praxis 3) und 15,3 Punkte (Praxis 2).

\section{Nebenwirkungen}

Die Verträglichkeit und mögliche Nebenwirkungen wurden regelmäßig abgefragt. Bei keinem Patienten zeigten sich Symptome, die als Nebenwirkung der Procain-Infusion hätten interpretiert werden müssen. Keine der insgesamt weit mehr als 1000 Infusionen, die im Rahmen dieser AWB appliziert wurden (d.h. einschließlich der Patienten, die im Rahmen dieser AWB zwar therapiert wurden, aber nicht in die AWB eingeschlossen werden konnten), musste wegen Unverträglichkeit abgebrochen werden.

\section{Diskussion}

Die vorliegende AWB ging der Frage nach, ob die intravenöse Gabe von Procain (Infusionen in $\mathrm{NaCl}$ ) bei chronischen psychovegetativen Störungen bzw. Störungen der Stressachse (Begriffe verwendet entsprechend gängiger Definitionen [15]) zu einer langanhaltenden Verbesserung führt. Verwendet wurde ein validierter Fragebogen zur Befindlichkeit (BSKE 30). Die Auswertung der anonymisierten Fragebögen ergab eine langanhaltende Verbesserung über 4 bzw. 6 Monate der Skala der positiven Items bei 42 Patienten $(75 \%)$ einerseits und der Skala der negativen Items bei 35 Patienten $(62,5 \%)$ andererseits.

Intravenöses Procain wurde früher oft in der Schmerztherapie appliziert, und noch heute ist der intravenöse Bolus von $1 \mathrm{ml}$ 1\%igem Procain ein fester Bestandteil der Neuraltherapie nach Huneke $[1,2]$. In der vorliegenden AWB wurde die eigenständige Wirkung einer alleinigen Gabe von intravenösem Procain unabhängig von einem Therapiekonzept wie der Neuraltherapie untersucht. Das schließt natürlich nicht aus, dass das intravenöse Procain mit seinen neurobiologischen Effekten zur Wirkung solcher Therapieverfahren beiträgt.

\section{Studiendesign und die Bedeutung von \\ Anwendungsbeobachtungen}

Diese AWB folgte einem unkontrollierten Ansatz. In Deutschland erhalten jährlich viele Tausende intravenös Procain, sei es als Bolus oder als Infusionen, sei es im Rahmen der Neuraltherapie oder als alleinige Gabe unabhängig von einem Therapiekonzept. Die Anwender haben in ihrem Praxisalltag immer wieder die geschilderten therapeutischen Wirkungen von Procain erlebt, über die vielfach auf Kongressen und Fortbildungen berichtet wird, und viele Patienten bitten - oftmals aufgrund früherer positiver Erfahrung - um Procain-Infusionen. In einem solchen Kontext, noch dazu über 6 Monate, ist eine kontrollierte AWB z.B. durch die Infusion von $\mathrm{NaCl}$ ohne Procain unter den Bedingungen des Praxisalltags schwierig durchzuführen und unter Umständen ethisch problematisch [16]. Meta-Analysen konnten gerade im Hinblick auf alltäglich erfahrbare therapeutische Wirksamkeit zeigen, dass Beobachtungsstudien bzw. AWB ähnliche Ergebnisse erbringen wie kontrollierte Studien [17]. AWB sind für die klinische Praxis immer noch unentbehrlich; so wurde in der renommierten Zeitschrift «The Lancet» darauf hingewiesen, dass ein bedeutender Teil der für den klinischen Alltag wichtigen Erkenntnisse aus Erfahrungsberichten und AWB stammt [18]. Vor kurzem haben die USamerikanischen National Institutes of Health eine Kampagne zu DRRR von bekannten oder älteren Wirkstoffen gestartet, um deren therapeutisches Potenzial auszuschöpfen (https://ncats.nih.gov). Die intravenöse Gabe von Procain erfüllt genau die Kriterien für eine solche Reevaluierung der Wirkung sogenannter alter und längst zugelassener Arzneimittel. Diese AWB hat sich so gut wie möglich an den Kriterien der STROBE-Guidelines orientiert [12].

\section{Therapeutische Wirkung von Procain}

Intravenöses Procain entfaltet seine Wirkung nicht nur im peripheren und vegetativen Nervensystem, sondern auch im Gehirn. Nach der Penetration durch die Bluthirnschranke erhöht Procain die Durchblutung, und damit die neuronale Aktivität, in verschiedenen zentralnervösen Kerngebieten wie dem präfrontalen Kortex, den Basalganglien und dem limbischen System [7-9]. Nachgewiesen sind unter anderem auch die Hemmung des DAT und die Zunahme der Verfügbarkeit von Dopamin im synaptischen Spalt [56] sowie die Hemmung der Serotonin- und Noradrenalin-Wiederaufnahme in vitro [19].

Die neurobiologischen Effekte von Procain, die unabhängig von seiner lokalanästhetischen Wirkung gesehen werden müssen, überraschen nicht angesichts seiner funktionellen und strukturellen Verwandtschaft mit Cocain, dessen neurobiologische Effekte auf die Systeme der biogenen Amine bestens bekannt sind. In Dosierungen, die den in dieser AWB verwendeten entsprechen, bewirkt Procain bei gesunden Menschen eine Unruhe und negative Stimmung, jedoch nicht bei ehemaligen Cocainabhängigen [7, 9]. Dies deutet darauf hin, dass Procain einen möglichen hypodopaminergen Zustand - den auch Patienten mit chronischen Schmerzen aufweisen - zu bessern vermag.

Hingewiesen sei auch auf die Anti-Tumor-Effekte von Procain, die ebenfalls unabhängig von der lokalanästhetischen Wirkung entstehen [20, 21], sowie auf die vasodilatatorische Wirkung des Procain-Metaboliten Diethylaminoethanol.

Ausmaß der Symptomverbesserungen durch Procain-Infusionen

Als Verbesserung wurde die Zunahme des positiven bzw. die Abnahme des negativen Gesamtscores um +7 (bei 54 möglichen Punkten) bzw. -10 Punkte (bei 72 möglichen Punkten) angenommen. In zahlreichen pharmakologischen Studien zur Arzneimittelwirkung gilt eine solche quantitative Veränderung von 13\% des Maximalwerts als signifikant, z.B. beim Hamilton-DepressionsScore oder bei der visuellen Schmerz-Analogskala. Die Effektstärke gegenüber dem Ausgangswert war bei den positiven bzw. netativen Items zu 3 von 4 bzw. allen 4 Beobachtungszeitpunkten mit $>0,5$ hoch. 


\section{Wer profitiert?}

Die Patienten dieser AWB zeigten vielfältige Krankheitssymptome. Ein gemeinsamer Nenner waren psychovegetative Störungen, wobei affektive oder schmerzhafte Symptome hervorstachen. Wer profitiert von Procain-Infusionen? Die Einschätzung der 3 Therapeuten lautet übereinstimmend: Es profitieren Patienten mit chronisch-entzündlichen Erkrankungen oder mit depressiv-affektiven Verstimmungszuständen. Wir deuten die Wirkung von Procain auch als Hilfe bei der Normalisierung von Regulationsstörungen bzw. von psychovegetativer Dysbalance, im Sinne eines Anstoßes zur Selbstheilung [22].

\section{Procain-Infusion ohne Basenzusatz}

Häufig werden Procain-Infusionen mit einem Basenzusatz in der Annahme appliziert, dass Natrium-Bicarbonat die Wirksamkeit von intravenösem Procain verbessert. Studien, die dies wissenschaftlich belegen, stehen aus. Die vorliegende Wirkung von Procain-Infusionen ist wahrscheinlich ein zentralnervöser Effekt, der sich unabhängig von einer begleitenden Bicarbonat-Gabe realisiert.

Die Diskussion über die Wirkung von Procain-Infusionen ohne Bicarbonat muss klar von der Frage getrennt werden, ob Bicarbonat eine eigenständige therapeutische Wirkung hat. Die Möglichkeit, dass Bicarbonat die Pharmakokinetik einer intravenösen Procain-Infusion, wie die Penetration durch die Bluthirnschranke oder die Verteilung im Gehirn, nennenswert beeinflusst, kann ausgeschlossen werden.

\section{Verträglichkeit}

Die wissenschaftliche Diskussion um eine therapeutische Wirkung von intravenösem Procain ruft bei vielen «Procain-naiven» Kolleginnen und Kollegen sofort einen Ablehnungsreflex hervor, der auf einer gefühlten Gefahr durch ein systemisch verabreichtes Lokalanästhetikum beruht. Der systemische therapeutische Effekt, wahrscheinlich durch die Modulation der biogenen Amine (vor allem Dopamin) bedingt, ist jedoch unabhängig von der lokalanästhetischen Wirkung zu sehen.

In den 1950er Jahren kam es zu gehäuften Zwischenfällen unter Procain mit dem Zusatz von Adrenalin im Rahmen operativer Eingriffe. Es ist das Adrenalin, das die Toxizität von Procain um das 2-3-fache erhöht und über eine eigene Toxizität verfügt [23].

Auch die Besorgnis hinsichtlich einer sogenannten Paragruppen-Allergie, die seit 60 Jahren durch die Tradierung unbewiesener Vermutungen am Leben erhalten wird, besitzt keine klinische Relevanz [23]. Im Rahmen dieser AWB mit mehr als 1000 Procain-Infusionen trat keine relevante Störung auf, die als ernste Nebenwirkung hätte interpretiert werden müssen. Damit bestätigt auch diese AWB die sehr gute Verträglichkeit von Procain, wie sie in den letzten mehr als 60 Jahren in zahlreichen Publikationen über die nebenwirkungsfreie intravenöse Anwendung hunderttausender Procain-Infusionen beschrieben wurde [1, 24, 25]. Als relevante Nebenwirkungen von Procain werden in den Fachinformationen ein vorübergehender Abfall des Blutdrucks, allergische
Reaktionen und schwere zentralnervöse Reaktionen wie Krampfanfälle erwähnt.

\section{Stärken und Schwächen der Anwendungsbeobachtung}

In dieser AWB wurde zum ersten Mal die Wirkung von Procain-Infusionen über bis zu 6 Monate in einem standardisierten Design untersucht. Das vordefinierte Protokoll wurde 3 Ethikkommissionen vorgelegt, und der validierte Fragebogen erfragt wichtige Items zur psychovegetativen Befindlichkeit, die einen Therapieerfolg messen können. Die 3 Arztpraxen sind über Süd-, Mittelund Norddeutschland verteilt und repräsentieren eine Patientenpopulation aus 1 Großstadt und 2 Mittelstädten. Eine Selektion nach positiver oder negativer Response fand nicht statt; alle Patienten, die die AWB begonnen hatten, wurden gebeten, mindestens 4 und nach Möglichkeit 6 Monate adhärent zu bleiben, was in 48 von 56 Fällen auch gelang.

Als Einschränkungen sind die Heterogenität der Teilnehmer, die nichtstandardisierte Gabe von Procain und fehlende Daten zur Häufigkeit der Procain-Infusionen und zur Anzahl der Behandlungen zu nennen. Jedoch muss auch hier der Praxisalltag als bestimmender Faktor berücksichtigt werden. Das Einschlusskriterium war die nach Ansicht der Therapeuten mögliche Responsivität der Patienten auf Procain; eine weitere Selektion fand nicht statt.

\section{Fazit}

Procain-Infusionen sind eine sichere und nebenwirkungsarme therapeutische Option, um somatische und psychovegetative Störungen bzw. Befindlichkeiten zu verbessern. Der zugrunde liegende, wahrscheinlich systemisch-zentralnervöse Effekt von Procain muss unabhängig von seiner lokalanästhetischen Wirkung gesehen werden. Dieses Ergebnis sollte durch doppelblinde randomisierte Studien abgesichert werden.

\section{Danksagung}

Der Autor J.D.H.-G. dankt seiner Mitarbeiterin Frau Corinna Wiegand für ihr engagiertes Mitwirken bei der Durchführung der Studie. Dank gilt auch Frau Dr. Silke Szymczak für ihre Unterstützung bei den statistischen Berechnungen.

\section{Disclosure Statement}

Die Autoren erklären, dass keine Interessenkonflikte vorliegen. Die AWB wurde gefördert durch den Förderverein Neuraltherapie e.V. 


\section{Literatur}

1 Barop H: Taschenatlas der Neuraltherapie nach $\mathrm{Hu}$ neke, ed 4. Stuttgart, Haug, 2017.

2 Fischer L: Neuraltherapie: Neurophysiologie, Injektionstechnik und Therapievorschläge, ed 4. Stuttgart, Haug, 2014.

3 Hahn-Godeffroy JD: Procain in der Neuraltherapie nach Huneke - Literaturübersicht und zusammenfassende Bewertung. Allgemeinarzt 1993;15:876-883.

$\checkmark 4$ Benson BE, Carson RE, Kiesewetter DO, et al: A potential cholinergic mechanism of procaine's limbic activation. Neuropsychopharmacol 2004;29:1239-1245.

5 Wilcox KM, Paul IA, Ordway GA, et al: Role of the dopamine transporter and the sodium channel in the cocaine-like discriminative stimulus effects of local anesthetics in rats. Psychopharmacol 2001;157:260-268.

6 Wilcox KM, Kimmel HL, Lindsey KP, et al: In vivo comparison of the reinforcing and dopamine transporter effects of local anesthetics in rhesus monkeys. Synapse 2005;58:220-228.

7 Adinoff B, Devous MD, Best S, et al: SPECT following intravenous procaine in cocaine addiction. Ann N Y Acad Sci 1999;877:807-810.

$\checkmark 8$ Adinoff B, Devous MD, Best SE, et al: Dose-response measures of $\mathrm{rCBF}$ and subjective changes following procaine in healthy female volunteers. Psychiatry Res 2002;114:123-135.
9 Adinoff B, Devous MD Sr, Cooper DC, et al: Neural response to lidocaine in healthy subjects. Psychiatry Res 2009;173:135-142.

10 Wernze H: Neue Ansätze bei der Krankenbefragung Medizinische Welt 1996;47:41-45.

11 Wernze $\mathrm{H}$, Herdegen T: Long-term efficacy of spironolactone on pain, mood, and quality of life in women with fibromyalgia: an observational case series. Scand J Pain 2014;5:63-71.

12 von Elm E, Altman DG, Egger M, et al: The Strengthening the Reporting of Observational Studies in Epidemiology (STROBE) statement: guidelines for reporting observational studies. Ann Intern Med 2007;147:573577.

13 Saager L: Erholung und Befinden nach aorto-koronarvenösen Bypass Operationen. Promotionsarbeit, Universität Lübeck, 2009.

14 Rosenthal JA: Qualitative descriptors of strength of association and effect size. J Soc Serv Res 1996;21:37-59.

15 Möller HG, Laux G, Kapfhammer HP (eds): Psychatrie, Psychosomatik, Psychotherapie, vol 2, ed 4. Berlin, Heidelberg, Springer, 2011, pp 791-801.

16 Glasziou P, Chalmers I, Rawlins M, et al: When are randomised trials unnecessary? Picking signal from noise. Brit Med J 2007;334:349-351.
17 Concato J, Shah N, Horwitz RI: Randomized, controlled trials, observational studies, and the hierarchy of research designs. N Engl J Med 2000;342:1887-1892.

18 Horrobin DF: Effective clinical innovation: an ethical imperative. Lancet 2002;359:1857-1858.

19 Sato T, Kitayama S, Mitsuhata C, et al: Selective inhibition of monoamine neurotransmitter transporters by synthetic loal anesthetics. Naunyn Schmiedebergs Arch Pharmacol 2000;361:214-220.

20 Li YC, Wang Y, Li DD, et al: Procaine is a specific DNA methylation inhibitor with anti-tumor effect for human gastric cancer. J Cell Biochem 2018;119:2440-2449.

21 Ying B, Huang H, Li H, et al: Procaine inhibits proliferation and migration and promotes cell apoptosis in osteosarcoma cells by upregulation of microRNA-133b. Oncol Res 2017;25:1463-1470.

22 Hahn-Godeffroy JD: Procain-Reset: Ein Therapiekonzept zur Behandlung chronischer Erkrankungen. Schweiz Z Ganzheitsmed 2011;23:291-296.

23 Hahn-Godeffroy JD, Barop H: Zur Arzneimittelsicherheit von Procain. Dtsch Z Akupunktur 2011;54:28-29.

24 Graubard DJ, Peterson MC: The therapeutic uses of intravenous procaine. Anesthesiology 1949;10:175-187.

25 Reuter U, Oettmeier R: Die hochdosierte ProcainBasen-Infusion. Ärztezeitschr Naturheilverf 1999;11: 776-782. 